\title{
Représentation discursive des femmes dans la production cinématographique au Cameroun
}

Carole FOPA ${ }^{1}$

\begin{abstract}
The presence of women in Cameroonian cinema begins from its origin. However, this presence is conditioned by men who define their role within this ecosystem. This article is aims at describing the discursive constructs produced by men on women within this system by portraying how they participate in its domestication and how these discursive products impact on filmmaking activities.
\end{abstract}

Keywords: Cameroon movies; ideology; discursive strategies; gendered discourses; stereotypes

DOI: $10.24818 / D L G / 2021 / 38 / 11$

\section{Introduction}

E

n 1970, le cinéma camerounais pousse officiellement son premier cri, la production du premier long métrage, Une nation est née de Jean Paul Ngassa marque la création d'un univers cinématographique endogène. Dans ses premiers pas, les rennes sont aussitôt tenus par les hommes. Une évidence si l'on considère le cinéma camerounais comme fille du cinéma occidental (Barlet,1999). Le privilège de la connaissance (cinématographique) a été prioritairement concédée aux hommes (Tcheuyap, 2010). L'histoire du cinéma camerounais va s'écrire avec les hommes en arrière-plan tenant les caméras, et les femmes en premier plan, devant les caméras, jouant des rôles définis pour elles. Le positionnement des femmes au-devant des caméras, peut laisser entrevoir une mise en valeur de celles-ci, mais comme Momar Desiré Kane le précise bien (cité par Barlet, 2009) : «Si puissante que la femme paraisse, c'est à travers des notions créées par la conscience mâle qu'elle est saisie ».

Les femmes ont fait tardivement leur entrée dans le domaine de la production au Cameroun. La toute première maison de production

${ }^{1}$ Carole Fopa, étudiante en PhD à l'université de Dschang, Cameroun, davefopah@gmail.com 
féminine va voir le jour en 1996, Alternatives Productions de Yolande Ekoumou. En réalité, moins de $20 \%$ de femmes sont à la tête d'une maison de production au Cameroun ${ }^{2}$. Ce chiffre suscite une réflexion sur le défi à relever en tant que femme pour évoluer dans un système phallocratique. En effet, plusieurs travaux ont été consacrés à la situation de la femme dans le milieu cinématographique, en plaçant au centre de l'analyse la représentation des femmes dans les œuvres filmiques (Bolla, 1979; Barlet, 2009 ; Tcheuyap, 2010 ; Nyamkoh et Ngwa, 2021). Il est question d'opter pour une démarche méthodologique distincte avec pour spécificité l'analyse des trajectoires individuelles pour «défamiliariser» les identités de genre et le binarisme qui les sous-tend, afin de rendre compte des opérations par lesquelles elles sont reproduites voire déplacées. Cet article a pour objectif de faire l'analyse discursive d'une catégorie «oubliée » de l'industrie du cinéma au Cameroun. Il s'agit spécifiquement de leur donner la parole et d'identifier dans leurs manifestations langagières comment elles représentent le monde dans lequel elles vivent. Comment l'idéologie de la «classe dominante" du cinéma se manifeste-t-elle dans leur discours ? Et comment ces représentations discursives impactent-elles la façon dont elles pratiquent la production?

La particularité de cette analyse est qu'elle s'appuie sur les récits des productrices pour examiner leurs rapports au masculin, la façon dont les représentations stéréotypées peuvent contribuer à limiter l'accès des femmes aux instances clés de l'industrie du cinéma au Cameroun. Nous avons opté pour l'examen des trajectoires individuelles parce qu'elles peuvent constituer des clés dans la compréhension des différentes représentations des femmes dans le milieu. En plus, cet article se propose également de mettre en lumière l'impact de ces représentations dans la construction d'une « identité auto-désigné ».

Le corpus que nous avons entrepris d'examiner dans cet article provient d'une large étude que nous avons menée sur les femmes dans l'industrie cinématographique au Cameroun de 2016 à 2018. Les entretiens avaient été enregistrés puis transcrits pour exploitation. Pour cet article,

2 Cette donnée a été obtenue à partir des sites (www.cinecamer.com; http://africultures.com www.africine.org). Nous avons fait une triangulation des données pour obtenir à partir des maisons de productions répertoriées sur l'ensemble du territoire camerounais la proportion des femmes à la tête de ces maisons de productions. 
nous nous sommes focalisés sur les questions adressées aux productrices principalement celles qui se réfèrent aux contraintes liées à leur métier de productrice et à leurs rapports aux hommes dans le métier.

Notre corpus se compose donc des entretiens de cinq productrices qui ont déjà produit ou coproduit un long métrage et qui sont à la tête d'une maison de production depuis plus de cinq années. Ces critères ont été définis avant la collecte tout simplement parce que nous les avons jugés nécessaires et même utiles pour comprendre les manifestations et même l'évolution (s'il en existe) des stéréotypes sur les femmes dans la production. Par ailleurs, le choix de collecter le témoignage individuel, repose surtout sur les raisons évoquées par Barlet (2009) : «le témoignage intime ouvre à une vision aporique ne donnant pas les solutions mais révélant les contradictions et les tensions ».

\section{Considérations théoriques}

Pour analyser la façon dont les femmes sont discursivement représentées dans la production cinématographique au Cameroun, nous avons choisi d'utiliser l'analyse critique du discours (ACD) pour découvrir comment le discours négocie et maintient les femmes « invisibles » dans la chaine industrielle cinématographique. L'usage de l'analyse critique du discours « présuppose un différentiel de pouvoir, c'est-à-dire qu'un groupe est relativement marginalisé au sein d'un pouvoir asymétrique » (Fairclough, 2003). De plus, l'ACD permet aussi de découvrir les hypothèses idéologiques qui sont cachées dans les mots de certains textes oraux (Fairclough, 1995 a). En tant qu'approche, elle a été utilisée pour nous permettre de mettre le doigt sur toutes les formes de stigmatisation et discrimination, mais surtout de relever le fait que le langage peut servir d'outil de dissuasion, voire d'entrave à la multiplication et à l'accession des femmes dans les instances clés de la chaine industrielle.

L'approche historique du discours a été retenue comme méthode d'analyse, pour faciliter l'identification des stratégies discursives qui participent à la subjectivité féminine. L'approche historique du discours suppose une relation dialectique entre des pratiques discursives particulières et des champs d'action spécifiques et tente de manière cruciale "d'intégrer une grande partie des connaissances disponibles sur les sources historiques et l'arrière-plan des champs sociaux [...] dans lesquels les événements «discursifs » sont enchâssés » (Reisigl et Wodak 2001 : 35). 
Ainsi, l'ACD a facilité le repérage textuel des éléments constitutifs de la construction subjective de l'identité féminine dans l'univers du cinéma au Cameroun et des motifs argumentatifs qui la sous-tendent.

\section{Cadre méthodologique ou analytique}

Dans la présente étude, nous nous sommes appuyés sur les outils développés par l'analyse critique du discours pour examiner les matérialisations linguistiques et rhétoriques visibles dans le discours féminin pour extérioriser toutes formes de représentations stéréotypées. Il s'est agi précisément d'opérer à l'analyse des données à partir de l'approche historique du discours (Wodak 2001 ; Reisigl et Wodak 2001 ; Weiss et Wodak 2003) pour faire une mise en relation des pratiques linguistico-discursives avec les pratiques sociales réelles (Petitclerc, 2014). A cette approche, nous avons associé, l'identification du discours ("Discourse identification») de Sunderland (2004:28) à travers une «lecture attentive» des marqueurs linguistiques dans le discours. En effet, les discours sont également identifiés à travers des " traces » linguistiques particulières qui sont des formes ou des «marques sur la page » (Talbot 1995 : 24). Les traces linguistiques pertinentes peuvent être lexicales ou syntaxiques (noms, verbes et adjectifs), des mots ou des phrases saillantes ou souvent répétées dans le discours (Atanga, 2012; Sunderland, 2004 ; Fairclough, 2003 : 124). Plus concrètement, ces traces linguistiques concernent les choix pronominaux, les formes syntaxiques (adverbes, interrogatifs, exclamatifs, structures phrasiques), (Atanga, 2012).

Pour cet article, nous avons commencé par identifier les «naming discourses ", les discours nominatifs (Atanga, 2012) qui participent aux construits discursifs sur les productrices dans le cinéma au Cameroun. Il s'agit des modèles de dénomination qui renforcent les structures idéologiques cachées dans la mémoire des peuples (Wodak, 2001). Ces modèles participent à la construction d'un "discours d'identité » sur les productrices dans le milieu cinématographique, qui est une identité attribuée, non auto-assignée (Atanga, 2012). Ensuite, nous avons identifié les stratégies référentielles et argumentaires déployées par ces femmes pour se créer leur propre identité et justifier leur « soi ». 


\section{Analyse des productions discursives}

\subsection{Discours de domestication des femmes : Sois belle et tais-toi !}

Il y a une évolution conceptuelle de la domestication féminine et cela intègre plusieurs unités de significations à l'instar de celle décrite par Atanga (2012). Elle ne réfère plus seulement à la gestion des tâches ménagères: "Whereas domestic in the past meant not having paid jobs and career women being greatly discouraged, recent social and economic developments (poverty, urbanisation and modernisation, globalisation (including global discourses on women's rights and the empowerment of women), greater perceptions of material needs), families often seek or need a second pay cheque»3 (Atanga, 2012:26). Ce besoin a conduit à une nouvelle forme de domestication qui induit une redéfinition et une réinterprétation du terme "domestique » principalement dans le contexte camerounais, et ce en lien avec les femmes (Atanga, 2012: 26). Autrement dit, il s'agit d'une « resémantisation » (Rabatel, 2001) du vocable «domestication » qui, dans le contexte de l'industrie du cinéma est la restriction et le maintien des femmes dans des rôles qui limitent leur participation dans les sphères de prise de décisions. La femme est conditionnée pour être une actrice et la logique masculine est de la maintenir dans ce statut :

[...], parce qu'étant productrice, elle risque ne pas être devant la caméra. En fait les hommes laissent libre cours aux femmes dans le cinéma camerounais pour certains rôles, actrice oui, mais quand c'est déjà le prestigieux poste de réalisatrice ou de productrice ça devient carrément autre chose. (Lucie Memba, productrice)

Le syntagme «les hommes laissent » dévoile une infantilisation et un contrôle sur la destinée de la femme. Cela sous-entend que les décisions de la femme en termes de ce qu'elle doit devenir dans le secteur cinématographique sont conditionnées par le masculin. En plus, l'adjectif indéfini "certains" apposé au nom " rôles" met bien en évidence cette volonté de domestication. Le destin de la femme dans ce milieu semble

\footnotetext{
${ }^{3}$ Alors que domestique dans le passé signifiait ne pas avoir d'emplois rémunérés et que les femmes de carrière étaient fortement découragées, les développements sociaux et économiques récents (pauvreté, urbanisation et modernisation, mondialisation y compris les discours mondiaux sur les droits des femmes et l'autonomisation des femmes ont renforcé la perception des besoins matériels), les familles recherchent ou ont souvent besoin d'un deuxième chèque de paie.
} 
bien défini. L'utilisation de l'adjectif « prestigieux » connote l'existence des catégories ou des strates au sein de la structure industrielle. Rappelons une fois encore que, cette analyse discursive a pour but, de mettre en évidence la façon dont la femme se représente la structure cinématographique, la façon dont elle perçoit ses rapports au masculin au sein de cette structure industrielle. Une stratification qui renvoie une image subalternisée de la femme, une personne qui doit constamment être reléguée au second plan :

Les femmes ont le talent. Les femmes ont naturellement le talent, si vous pouvez être mère, épouse, entrepreneur c'est que vous avez naturellement le talent mais c'est à cause de l'égo masculin. Que ce soit au Cameroun en Afrique ou en Occident partout on s'arrange à ce que la femme soit mise à la place qu'ils veulent qu'elle soit mais jamais à la place qu'elle aimerait. Et ce n'est pas parce que les femmes n'ont pas le talent, les femmes en ont énormément mais on ne leur permet pas et dans le cinéma c'est la même chose, les hommes ne permettent pas! (Flavienne Tchatat, productrice)

L'usage du pronom personnel indéfini «on » a pour référentiel les hommes. Il y a deux occurrences dans cet extrait discursif. Ce pronom bien qu'il soit indéfini, il apparaît dans ce contexte dans sa forme généralisatrice et exclut d'emblée la locutrice. Il a pour référentiel tous les hommes qui exercent le contrôle et le pouvoir sur la femme en tentant quotidiennement de la réduire au rôle de domestique.

Dans la phrase exclamative "les hommes ne le permettent pas !", l'unique marqueur morphosyntaxique est le point d'exclamation qui l'accompagne. En réalité, pour la ranger comme phrase exclamative nous avons fait appel aux traits acoustiques qui s'y dégageaient au moment de l'enregistrement pour saisir l'intonation de la voix de la locutrice. Cette phrase exclamative permet de saisir au vif l'émotion qui la parcourt. Par ailleurs, elle permet de saisir l'environnement et le climat dans lequel les femmes évoluent dans le milieu cinématographique. Cet environnement que nous pouvons caractériser d'hostile a pour but d'emprisonner la femme dans une destinée figée (Beauvoir, 1949) : celle de demeurer actrice. De plus, l'emploi du verbe "permettre" au présent de l'indicatif situe l'action dans le présent et donne à penser que les actions d'hostilité envers les femmes pour les maintenir dans leur condition de «domestique » ne sont pas des actions dépassées, elles sont bien actuelles pour que «la femme soit mise à la place qu'on voudrait qu'elle soit ». Cette deuxième phrase montre de toute évidence qu'il y a une domestication de la gent 
féminine, car, à l'image d'un animal, dompté, dressé et domestiqué on attend de la femme cinéaste qu'elle rentre dans la « case » qui est sienne.

\subsection{Discours de la fragilité des femmes}

Pour énoncer le discours sur la fragilité de la femme, nous avons choisi de planter le décor, en convoquant le digeste, un recueil de jurisprudence classique publié en 533 qui évoque un assortiment de mots en latin pour illustrer la faiblesse naturelle de la femme: infirmitas, imbecillitas et fragilitas (Beaucamp, 1994). Ces mots épars réfèrent à une signification unique, la fragilité de la femme qui la rend inapte à accomplir certaines tâches à raison de son sexe (Beaucamp, 1994). Dans le même texte, apparait une occurrence flagrante des mots se rapportant à la fragilité et qui démontre comment: «[...] (cette) faiblesse empêche aussi les femmes de mettre en mouvement certaines procédures judiciaires » (Beaucamp, 1994). Un ensemble de traits singuliers liés à son sexe qui tend à l'enfermer dans sa subjectivité : "La femme a des ovaires, un utérus ; voilà des conditions singulières qui l'enferment dans sa subjectivité ; on dit volontiers qu'elle pense avec ses glandes.» (Beauvoir, 1949) Cette singularité féminine constamment relevée par les hommes apparaissent comme des contraintes pour la femme : «Les vraies contraintes sont qu' on a tendance à vous traiter (les hommes sont comme ça naturellement) d'une certaine manière. » (Augustine Moukodi, productrice)

Dans ce discours de la productrice Augustine Moukodi, l'article indéfini «une» qui accompagne le syntagme adverbial "certaine manière » connote la mise en index de la femme, ce sur la base de son appartenance sexuelle. En outre, la locution adverbiale «certaine manière » traduit également le traitement différentiel que subit les femmes dans l'industrie du cinéma, conséquence de la socialisation différentielle des sexes (Mosconi, 2010). Dans le cadre de la production du film, Augustine Moukodi nous raconte le récit de la production du film our wishes (2017) et le discours articulé autour de sa condition de femme pendant tout le processus :

Mais ça créait parfois la surprise chez beaucoup de gens que j'ai rencontrés justement. Certains même me proposaient un boulot dans un bureau par exemple. Est-ce que ce n'est pas difficile ce que vous avez choisi de faire ? La production. Pour la petite histoire, à Zili la route est tellement mauvaise si bien que tout le temps la voiture avait des problèmes. Et donc, il fallait prendre des motos et parfois on tombait sur 
des gars qui ne maitrisaient pas forcément la route et donc, il arrivait qu'on tombe. Donc, on avait acheté un casque, quand je partais de Zili pour aller au ministère j'avais mon casque en main, j'entrais dans le bureau du Directeur de la cinématographie avec mon casque, on me demandait vous avez une moto et je lui disais non et je lui racontais l'histoire du casque et on me demandait pourquoi est-ce que vous vous mettez dans ce genre de chose? pourquoi vous ne trouvez pas un boulot simple?

La phrase interrogative «Est ce que ce n'est pas difficile ce que vous avez choisi de faire ? » semble traduire l'inquiétude de l'interlocuteur qui de toute évidence est de sexe masculin. Cette phrase interrogative revêt une autre connotation. Car, si la question de la difficulté a été posée à la productrice, il est évident que c'est en rapport avec son sexe et les stéréotypes qui l'entretienne. En d'autres termes, ce discours aurait certainement été différent s'il s'agissait d'un homme. Pour la simple raison que l'attribut de force semble avoir été conféré «naturellement » à l'homme en opposition à la faiblesse et à la fragilité que semble revêtir le sexe féminin. L'adjectif qualificatif «difficile» présent dans cette phrase interrogative vient renforcer le discours articulé autour de la fragilité de la femme.

De plus, la phrase «Certains même me proposaient un boulot dans un bureau par exemple » légitimise ce discours de la fragilité de la femme. Car, au Cameroun, le travail de bureau est perçu comme moins pénible et moins difficile à exécuter et par conséquent plus adapté pour les êtres fragiles que sont les femmes. Par ailleurs, les deux phrases interrogatives "pourquoi est-ce que vous vous mettez dans ce genre de chose?", " pourquoi vous ne trouvez pas un boulot simple? » concluant le discours de Augustine Moukodi sont sémantiquement chargées de stéréotypes sur ses capacités physiques et professionnelles. Elles sont empreintes d'une connotation stéréotypée sur le métier de production qui semble être socialement façonné pour les hommes. Ceci dans la mesure où, dans la sphère professionnelle, les femmes sont assignées à certaines catégories socioprofessionnelles. Et de manière générale, la division sexuée du travail postule que : «habileté et concentration sont des qualités professionnelles dites féminines, là où la force physique et morale, l'autorité et l'autonomie sont des caractéristiques dites masculines par excellence » (Missoffe, 2015). 


\subsection{Femme comme objet sexuel}

La femme-objet est un discours de marchandisation et d'instrumentalisation $\mathrm{du}$ corps de la femme. Il s'agit aussi de l'appropriation sexuelle des femmes par les hommes. Dans le milieu cinématographique, principalement celui de la production, la mobilisation des ressources matérielles, humaines et même financières pour la réalisation d'un film au Cameroun relève d'un véritable tour de magie. La situation embryonnaire de l'industrie du cinéma au Cameroun rend plus complexe la situation de la femme. Les femmes dans le domaine cinématographique sont prises pour cible par les hommes qui les perçoivent comme l'objet de leur désir :

Ça a été compliqué et jusqu'aujourd'hui ça reste toujours compliqué parce que si j'ai voulu abandonner le cinéma pour la première fois c'est parce que mon formateur M. [...] m'avait fait la cour et c'était pour moi un choc déjà j'étais jeune, venir là pour faire les formations pour essayer de réaliser mes projets et puis sans même y arriver le formateur me fait la cour, pour moi ça été un choc. (Sandrine NGueffo, productrice)

Dans le discours de cette productrice qui nous raconte les récits de ses premiers pas dans le monde cinématographique, on peut déduire à travers l'usage de l'euphémisme «faire la cour» qu'elle aurait hypothétiquement été harcelée. L'usage de la phrase «J'ai voulu abandonner » confirme cette hypothèse du harcèlement sexuel. Il y a de toute évidence une forte pression sexuelle autour des femmes. Cette pression sexuelle a conduit à une représentation objectivée de la femme. En réalité, il y a une forte représentation discursive autour du succès des productrices, un discours qui réduit son activité à la marchandisation de son corps :

C'est vraiment très compliqué mais de toutes les façons la femme qui évolue est toujours vue comme celle qui a donné son corps pour atteindre un niveau on ne cherche pas à voir le talent, les nuits de travail, tout ça est réduit à un seul mot la sexualité et c'est ça le problème. (Lucie Memba, productrice)

Cette phrase métaphorique "celle qui a donné son corps pour atteindre un niveau » employée par cette productrice donne une claire perception des représentations des productrices à succès au Cameroun. Cette comparaison implicite des productrices aux travailleuses de sexe traduit clairement la représentation populaire des femmes dans le milieu cinématographique. Les productrices marchandes leurs corps, ce sont des 
objets, des femmes incapables de tout accomplissement, dont le succès repose sur la commercialisation de leurs corps. Voilà donc le discours qui accompagne la réussite d'une productrice dans le milieu du cinéma : leur succès est inéluctablement rattaché à l'homme.

\section{Représentation discursive et pratique cinématographique : la justification du soi}

Les représentations discursives sur les productrices dans le cinéma au Cameroun impactent sur leur façon d'envisager le monde et le soi. Pour exister elles doivent perpétuellement déconstruire, décoloniser, négocier de façon continue leur identité « en subvertissant et en remettant en question les stéréotypes qui cherchent à justifier leur identité » (Atanga, 2020). Ici, il est question d'analyser les stratégies discursives employées par les productrices pour légitimer leur existence et affirmer leur identité. Les stratégies discursives renvoient aux différentes dimensions textuelles (Atanga, $2010: 34)$ ou comme Wodak (2001 :73) écrit : « [aux] différents niveaux d'organisation linguistique et de complexité ». Donc, il est question de focaliser notre attention sur les stratégies rhétoriques déployées par les productrices camerounaises pour justifier leur soi.

Les stratégies rhétoriques se rapportent à la capacité d'utiliser la langue efficacement et de manière convaincante (van Dijk, 2006 : 378). Cela peut être fait par le biais de l'utilisation d'hyperbole, l'expression d'émotions, métaphores, proverbes, analogies et jeux de mots (Atanga, 2012). La productrice camerounaise pour se construire une identité, déconstruit les stéréotypes et les préjugés rattachés à sa condition de femme, à son sexe.

C'est très courant dans le domaine du cinéma et beaucoup d'hommes pensent que si Sandrine Gueffo aujourd'hui a atteint tel niveau, $c^{\prime}$ est parce qu'elle a dû donner son corps à tel personne mais ils ignorent le talent qu'il y'a derrière. Le travail que vous abattez jours et nuits, etc. donc le challenge est vraiment énorme énorme énorme, dans la ville de Douala je vous assure le challenge est vraiment énorme.

La répétition du mot «énorme » dans ce discours laisse entrevoir toute la difficulté qu'il y a à se bâtir une carrière dans ce milieu. Elle traduit également la conscience de la difficulté qu'il y a à déconstruire les préjugés autour de la femme. Le récit de Augustine Moukodi illustre parfaitement 
comment les stéréotypes impactent sur les pratiques cinématographiques des femmes au quotidien :

La vérité c'est que les femmes doivent travailler deux fois plus ou trois fois plus. Quand vous travaillez dans un projet avec tel, on pense que vous devez avoir une relation particulière, ce n'est pas forcément par rapport à vos compétences. En tant que femme, j'ai dû être un homme parfois pour pouvoir avancer en même temps si non beaucoup plus que les hommes avec qui je travaillais.

Pour déconstruire les représentations liées à leur condition d'être fragile et restaurer leur image, "les femmes doivent travailler deux fois plus ou trois fois plus». Cette phrase hyperbolique employée par cette productrice met en évidence l'impact des représentations sur leur métier de productrice. Elles doivent faire plus d'efforts par rapport aux hommes dans leur milieu pour que leur statut de femme ne soit pas entrevu comme un handicap. Par ailleurs, l'usage de la métaphore "j'ai dû être un homme " peut traduire cette volonté sous-jacente de se hisser au même niveau que les hommes, d'être prise autant au sérieux que les hommes, de se bâtir une identité et de justifier leur soi.

Donc si vous voulez vous en sortir et que l'homme avec qui vous travaillez vous respecte en tant que collègue et ne vous voir pas en tant que femme, vous devez faire plus que lui, c'est comme ça que vous gagnez le respect auprès des hommes. (Evodie Ngueyeli, productrice)

Dans les deux structures syntaxiques « vous respecte en tant que collègue ", " ne vous voit pas en tant que femme ». La comparaison utilisée dans les deux structures syntaxiques met en relief cette volonté de s'affirmer et de justifier son identité. Une volonté de ne pas laisser ses compétences professionnelles être résumées à son sexe.

\section{Conclusion}

Nous avons utilisé l'analyse critique du discours dans cet article pour démontrer les représentations discursives en cours sur les femmes dans le domaine de la production au Cameroun. Nous avons relevé dans les discours des femmes interviewées que les femmes étaient généralement représentées discursivement comme des êtres fragiles, des objets, des femmes à domestiquer. A partir de ces discours, les femmes ont trouvé des mécanismes pour justifier leur identité. Les productrices camerounaises doivent non seulement se frayer une voix, un chemin dans un univers à 
prédominance masculine mais également elles doivent faire face aux discours sexistes qui sont constamment produits sur elles. Ce «discours d'identité » qui façonne la femme au goût et à l'image de l'homme en l'objectivant de façon permanente endigue leur épanouissement réelle et totale dans cet écosystème.

Toutefois, la nécessité de déconstruire ces stéréotypes et de justifier le soi transparaît dans leurs manifestations discursives. Le besoin de faire surgir une identité auto-assignée qui permettrait de se définir comme productrice camerounaise, de s'affirmer auprès de ses pairs et collègues, sans que ses compétences, ni ses talents ne soient entachés par les stéréotypes liés à sa féminité, ce besoin est apparu dans tous les discours des femmes interviewées. Cela montre de toute évidence que, malgré les changements apparents, la condition de la femme ne connait pas dans le fonds une très grande évolution. Les idéologies traditionnelles continuent d'avoir une emprise forte sur la manière dont fonctionne le monde, et l'image de la femme dans ce monde n'a pas beaucoup évolué, elle demeure dans l'imaginaire collective, cet être fragile qu'il faut protéger, ou encore cet être mythique dont les valeurs ont été autrefois célébrées par Léopold Sédar Senghor : «Une mythisation que Simone de Beauvoir n'a de cesse de dénoncer comme expression ultime du pouvoir de l'homme» (Barlet, 2009).

\section{Références bibliographiques}

1. ATANGA, Lem Lilian (2012), "The discursive construction of a "model Cameroonian Woman" within the Cameroonian parliament » GL, vol.6.1 Equinox Publishing, pp. 21-46.

2. ATANGA, Lem Lilian, (2020) «A gendered academy - women's experiences from higher education in Cameroon», De Gruyther Mouton, pp. 28-42. https://doi.org/10.1515/ijsl-2020-0080

3. BARLET, Olivier (1996), Les Cinémas d'Afrique noire. Le regard en question, Paris, L'Harmattan.

4. BARLET, olivier, (2009) «La représentation de la femme dans les cinémas d'Afrique noire", Revue Africulture, N74 http://africultures.com/la-representation-de-la-femme-dans-lescinemas-dafrique-noire-8345/ 
5. BEAUCAMP, Joëlle (1994), «Discours et normes : la faiblesse féminine dans les textes protobyzantins ». In : Cahiers du Centre Gustave Glotz, 5, pp. 199-220; doi : https://doi.org/10.3406/ ccgg.1994.1390 https://www.persee.fr/doc/ccgg_1016-9008_1994_num _5_1_1390

6. BOLLA, Corinne (1979), "Cinéma : nouvelles images de la femme au cinéma, mythe ou réalité ? » Intervention, (5), 37-38.

7. DE BEAUVOIR, Simone, (1949), Le deuxième sexe I : les faits et les mythes, Paris, Gallimard.

8. FAIRCLOUGH, Norman (1995a), Critical Discourse Analysis: the critical study of language, London: Longman

9. FAIRCLOUGH, Norman (2003), Analysing Discourse: Textual Analysis for Social Research, London: Routledge.

10. MISSOFFE, Prune (2015), «Stéréotypes, représentations sexuées et inégalités de genre dans les manuels scolaires ", La Revue des droits de l'homme [Online], 8 । Online since 23 November 2015, connection on 14 March 2021. URL : http://journals.openedition.org/revdh/1667 ; DOI: https://doi.org/10.4000/revdh.1667

11. MOSCONI, Nicole (2010), "Effets et limites de la mixité scolaire », Travail, genre et sociétés, 2004/1 n 11, p. 165-174 // « Filles / garçons Éducation à l'égalité ou transmission de stéréotypes sexistes ? », L'école et la ville.

12. NYAMKOH WANYU, Ernest, et NGWA, Arnold (2021), «Ideology, Power and Gender Representation in two Selected Nollywood Movies. » African Social Science and Humanities Journal, 2(1), 1-11. Retrieved from https://journals.jfppublishers.com/asshj/article/ view/28.

13. PETITCLERC, Adèle (2014), «Le postulat critique au cœur de l'analyse de discours. Introduction critique aux bases méthodologiques et épistémologiques des Critical Discourse

14. Studies ». Linguistique. Université de Franche-Comté, Français. ffNNT : 2014BESA1017ff. fftel-01174101ff*

15. RABATEL, Alain (2001), «La valeur de « on » pronom indéfini/pronom personnel dans les perceptions représentées », In : L'Information Grammaticale, N. 88, pp. 28-32. doi : 10.3406/igram.2001.2726 http://www.persee.fr/doc/igram_0222-9838_ 2001_num_88_1_2726 
16. REISIGL, Martin, et WODAK, Ruth (2001), Discourse and Discrimination: Rhetorics of Racism and Anti-Semitism, London: Routledge.

17. SUNDERLAND, Jane (2004) Gendered Discourses. Basingstoke: Palgrave.

18. TALBOT, Mary, (1995) Fictions at Work: Language and Social Practice in Fiction. London : Longman.

19. TCHEUYAP, Alexis (2010), «Cinéma documentaire et expériences féminines en Afrique francophone ", French Forum, 35(2/3), 57-77. Retrieved March 27, 2021, from http://www.jstor.org/stable/41306662.

20. VAN DIJK, Teun (2006), Discourse and manipulation. Discourse and Society 17(3): 359-83. http://dx.doi.org/10.1177/0957926506060250

21. WEISS, Gilbert, et WODAK, Ruth (2003), Critical Discourse Analysis: Teory and Interdisciplinarity, Basingstoke: Palgrave.

22. WODAK, Ruth (1985), «The interaction between judge and defendant. » in VAN DIJK, Teun (Dir.), Handbook of Textlinguistics, Vol. IV. Cambridge : Academic Press, pp.181-192. 